\title{
Prevalencia de tabaquismo en pacientes de la consulta psiquiátrica
}

\author{
Ricardo H. Ghisays ${ }^{1}$, Christian A. Gómez², Adalberto Campo ${ }^{3}$, \\ Martín Suárez ${ }^{4}$, Adolfo Bermúdez ${ }^{5}$
}

\begin{abstract}
Resumen
Varios estudios han encontrado que la prevalencia del hábito de fumar es más alta entre los individuos con trastornos mentales que en la población general. El objetivo del estudio fue determinar la prevalencia de tabaquismo en pacientes psiquiátricos ambulatorios de la ciudad de Cartagena. Se utilizó una breve entrevista estructurada para investigar el hábito de fumar en una muestra de 239 pacientes, encontrándose que $15,1 \%$ de los pacientes fumaba regularmente; el mayor porcentaje de fumadores eran de género masculino $(p<0,005)$; los diagnósticos más relacionados con el fumar fueron los trastornos psicóticos $(p<0,03)$. Se concluye que la prevalencia de tabaquismo en pacientes con trastornos psiquiátricos es similar a la encontrada en la población general; además, se puede considerar que existe una relación entre los trastornos psicóticos y el hábito de fumar.
\end{abstract}

\section{Summary}

Several studies show that the use of tobacco, especially smoking, is most common among psychiatric patients. The objective of this study was to find smoking prevalence among psychiatric outpatients in Cartagena. Using a short structured interview, smoking tobacco prevalence was investigated in a 239-patient sample. We found that $15.1 \%$ patients usually smoke; most of them were male $(p<0.005)$; psychotic disorders diagnosis were more frequently associated with smoking $(p<0.03)$.

In conclusion, smoking prevalence in psychiatric outpatients is similar to that of the general population; moreover, there is a link between a diagnosis of psychotic disorder and smoking that must be further investigated.

El hábito de fumar ha sido identificado como la causa más importante de morbimortalidad previsible que afecta la población general (1, 2). Los efectos nocivos de la nicotina pueden hacerse evidentes en cualquier sistema del organismo, de forma directa o indirecta, encontrándose relación causal directa, entre otras, con el cáncer pulmonar (3-5).
Aunque la tendencia actual de fumar en la población general ha mostrado una tendencia hacia la disminución (6-9), la observación clínica indica que las personas con problemas para controlar su estado emocional son particularmente dadas al abuso y la dependencia de sustancias, entre ellas la nicotina $(10,11)$. De la misma forma, las per-

\footnotetext{
Profesor Asociado II, Departamento de Psiquiatría, Universidad de Cartagena, Cartagena.

2 Jefe, Servicio de Urgencias, Hospital San Pablo, Cartagena.

3 Residente Nivel III, Departamento de Psiquiatría, Universidad de Cartagena.

4 Profesor Asistente I, Departamento de Psiquiatría, Universidad de Cartagena.

5 Profesor Asistente I, Departamento de Psiquiatría, Universidad de Cartagena.
} 
sonas normales fuman un mayor número de cigarrillos cuando la situación es estresante o displacentera (12).

Evidenciando esta situación, se ha observado la existencia de una estrecha relación entre el hábito de fumar y los trastornos mentales, particularmente con los trastornos depresivos, la dependencia de alcohol y la esquizofrenia $(10,13-19)$. Esta relación observada es claramente bidireccional, primordialmente en el caso de los trastornos depresivos, pues, los pacientes con un humor deprimido son más propensos a fumar que la población general y, por otra parte, la nicotina induce episodios depresivos (20-22).

Sin embargo, otros autores sostienen que no existe relación causal nicotina-depresión, depresión-fumar y tal vez lo que existe es una influencia genética común sobre algunos sistemas fisiológicos que predisponen tanto a la depresión como al tabaquismo $(23,24)$; otros consideran que es necesario tener presente siempre las características de la personalidad del individuo (25).

Las pruebas cuantitativas confirman que los individuos fumadores alcanzan puntajes más altos que los no fumadores, cuando son evaluados con pruebas para ansiedad y para depresión (20). También se ha reportado que el $60 \%$ de los individuos fumadores deseosos de abandonar el consumo tienen antecedentes de trastornos depresivos mayores (20).

El estudio realizado por el Ministerio de Salud de Colombia (1993) sobre salud mental y consumo de sustancias psicoactivas, en el cual se entrevistaron 875 personas, 439 de sexo femenino y 436 de sexo masculino, muestra que la prevalencia actual de tabaquismo en la población general es del $17,6 \%$ en el departamento de Bolívar, en tanto que la prevalencia encontrada a lo largo de la vida fue del $26,3 \%$. El mismo estudio indica que la prevalencia del consumo en hombres duplica la observada en mujeres $(23,4 \%$ vs $11,8 \%)$, alcanzando una diferencia significativa $(p<0,00001)$ (6) (tabla 1).

El objetivo general de este estudio fue determinar la prevalencia del consumo de cigarrillos
Tabla 1. Prevalencia de tabaquismo según género.

\begin{tabular}{lrrrrr}
\hline Género/ fumar & Sí & $\%$ & No & $\%$ & Total \\
\hline Femenino & 52 & 11,8 & 387 & 88,2 & 439 \\
Masculino & 102 & 23,4 & 334 & 76,6 & 436 \\
Total & $\mathbf{1 5 4}$ & $\mathbf{1 7 , 6}$ & $\mathbf{7 2 1}$ & $\mathbf{8 2 , 4}$ & $\mathbf{8 7 5}$ \\
\hline
\end{tabular}

Fuente: Estudio nacional de salud mental y consumo de sustancias psicoactivas (departamento de Bolívar). Bogotá: Ministerio de Salud, 1994.

en una población de pacientes ambulatorios de Cartagena, con diagnóstico psiquiátrico, en dos instituciones que prestan servicio de consulta externa psiquiátrica y en la consulta privada de los investigadores. En el presente artículo se muestran los resultados obtenidos en los pacientes evaluados en la consulta privada.

\section{Método}

Se tomó una muestra de 239 pacientes de la consulta psiquiátrica privada de Cartagena para este estudio; el tamaño de la muestra se calculó con un intervalo de confianza del 95\%, una probabilidad de 0,5 de encontrar el problema y un margen de error muestral del $5 \%$.

Se incluyeron en el estudio pacientes de ambos sexos, mayores de 18 años, que llenaban los criterios para cualquier categoría diagnóstica de la clasificación de la Asociación Psiquiátrica Americana (DSM-iV) (26).

Los individuos se evaluaron mediante una entrevista clínica no estructurada para determinar el diagnóstico psiquiátrico actual (eje I). La evaluación relacionada con el hábito de fumar consistió en una breve entrevista estructurada. La pregunta que debían contestar afirmativamente para ser considerados fumadores fue: ¿Fuma usted actualmente?

Los diagnósticos psiquiátricos se agruparon en cuatro grandes categorías: trastornos de ansiedad (trastorno de ansiedad generalizada, obsesivo-compulsivo, etc.), trastornos del humor (trastornos depresivos, trastorno bipolar, etc.), trastornos psicóticos (esquizofrenia, 
trastorno esquizo-afectivo, etc.) y otros (abuso y dependencia de drogas, disfunciones sexuales, demencia, etc.).

Se utilizó el programa EPI-INFO 6.01 (CDC, OMS, 1994) para el almacenamiento y procesamiento de los datos. El análisis comparativo de los resultados se realizó con las pruebas de ji-cuadrado y $t$ de Student; se aceptaron como diferencias significativas, valores de $p<0,05$.

\section{Resultados}

Los resultados muestran que $121(40,6 \%)$ de los pacientes habían fumado alguna vez en su vida, mas solamente $36(15,1 \%)$ continuaban fumando en el momento de la evaluación.

El rango de edad del grupo osciló entre los 18 y los 81 años, con un promedio de 44,9 años (DS 14,5). La edad de los pacientes fumadores se encontró entre 19 y 59 años, un promedio de 40,5 años (DS 9,2), siendo significativamente menor comparada con la de no fumadores que tuvo una media de 45,6 años (DS 15,1) $(\mathrm{t}=11,25, \mathrm{p}<0,001)$.

De los 121 pacientes que alguna vez habían fumado, $117(96,7 \%)$ manifestaron que el inicio en el consumo fue previo a la consulta psiquiátrica comparados con $33(91,7 \%)$ de los que continuaban fumando regularmente que iniciaron el consumo previo al diagnóstico; sin embargo, la diferencia no fue significativa.

En relación con el género, $136(56,9 \%)$ de los pacientes entrevistados pertenecían al femenino y $103(43,1 \%)$ al masculino. Al comparar la relación entre el hábito de fumar actual y el género, se observó una diferencia significativa que mostraba una mayor prevalencia del fumar entre los hombres $(23,3 \%)$ comparado con las mujeres $(8,8 \%)$ [OR=3,14 (IC $1,41<\mathrm{OR}<7,10), \mathrm{X}^{2}=8,5 \mathrm{con}$ corrección de Yates, $p<0,005]$ (figura 1).

El nivel educativo demostró que, en promedio, el grupo había aprobado 10,1 años (DS 3,9). Se observó que no existía diferencia importante en los años de estudio de los fumadores y los no fumadores [10,5 (DS 3,8) vs. 10 (DS 3,9), $p>0,05]$.
Figura 1. Frecuencia de tabaquismo según género.

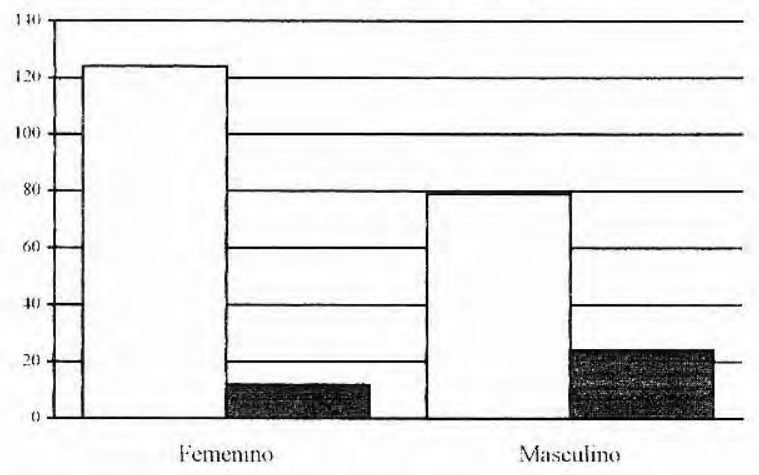

Dilerencra Signilicativa. $X_{2}=8.5$. Correceron de Yates $\left.p<0\right)(x(15$

$$
\text { № Fuma 国, riuma }
$$

En relación con el diagnóstico psiquiátrico actual, $148(61,9 \%)$ pacientes reunían criterios para un trastorno del humor; $38(15,9 \%)$ para un trastorno psicótico; $33(13,8 \%)$ para un trastorno de ansiedad y $20(8,4 \%)$ fueron incluidos en la categoría de otros; en este grupo, cinco pacientes presentaban un trastorno relacionado con el consumo de sustancias.

De la misma forma, fue evidente un predominio de fumadores actuales entre los pacientes con diagnóstico de un trastorno psicótico $(35,7 \%)$ en comparación con las otras categorías diagnósticas $\left(X^{2}=9,65, g l=3, p<0,03\right)$ (tabla 2).

Los pacientes con mayor prevalencia de tabaquismo durante la vida fueron, igualmente, los que fueron incluidos en la categoría de otros, lo cual está determinado por los trastornos relacionados con el uso de sustancias)

Tabla 2. Prevalencia actual de tabaquismo según diagnóstico.

\begin{tabular}{lrrrrr}
\hline $\begin{array}{l}\text { Fumar } \\
\text { Diagnóstico }\end{array}$ & Sí & $\%$ & No & $\%$ & Total \\
\hline Ansiedad & 3 & 9,1 & 30 & 90,1 & 33 \\
Humor & 17 & 11,5 & 131 & 88,5 & 148 \\
Psicóticos & 10 & 35,7 & 28 & 64,3 & 38 \\
Otros & 6 & 30,0 & 14 & 70,0 & 20 \\
Total & $\mathbf{3 6}$ & $\mathbf{1 5 , 1}$ & $\mathbf{2 0 3}$ & $\mathbf{8 4 , 9}$ & $\mathbf{2 3 9}$ \\
\hline
\end{tabular}

Diferencia significativa, $X^{2}=9,65, g l=3, p<0,03$ 
(75\%); sin embargo, la diferencia no alcanzó significancia estadística.

\section{Discusión}

Los resultados de este estudio muestran una prevalencia del hábito de fumar en pacientes psiquiátricos $(15,1 \%)$ comparable con la de la población general del departamento de Bolívar $(17,6 \%)(6)$.

Si consideramos la prevalencia según el género, los hombres con diagnóstico psiquiátrico fuman en similar porcentaje que los de la población general $(23,3 \%$ vs. $23,4 \%)$; aunque, la prevalencia de tabaquismo en las mujeres de la comunidad es ligeramente mayor a la observada en mujeres de este estudio $(11,8 \%$ vs. $8,8 \%)$, ésta no alcanzó diferencia significativa (6). Lo evidente es que el consumo de tabaco es más prevalente entre los hombres, tanto los que padecen trastornos mentales como en los de la población no diagnosticada $(5-6,12)$.

La prevalencia mostrada por este estudio difiere considerablemente de la observada en pacientes con trastornos mentales por otros autores que hallaron una prevalencia de tabaquismo mayor del $30 \%$, evidentemente mayor, que la observada en los estudios de la comunidad (14-15, 18).

En relación con el inicio del consumo, los resultados muestran que un $8,3 \%$ de los pacientes actualmente fumadores comenzaron el hábito de fumar después de la primera consulta psiquiátrica, aunque la misma es aproximadamente el cincuenta por ciento de la encontrada por otros autores (16\%) (12), la diferencia no alcanzó significancia estadística; sin embargo, es necesario tener presente que dicho estudio sólo incluía pacientes con esquizofrenia.

Los datos obtenidos, por tanto, no permiten precisar hasta qué punto el tabaquismo podría considerarse un síntoma, tal vez prodrómico, de un trastorno mental, como tampoco si su inicio, una vez realizado el diagnóstico, puede estar relacionado con el consumo de fármacos. Es probable que la nicotina sea utilizada por los pacientes ansiosos con el fin de disminuir la ansiedad y por los pacientes con trastornos psicóticos como droga para disminuir los efectos colaterales producidos por los neurolépticos usados en su tratamiento farmacológico (12, 14, 20-21, 27-28).

De otra parte, en este estudio observamos una alta prevalencia del uso del tabaco entre los individuos que consultaron por trastornos relacionados con sustancias; en relación con este tópico, algunos autores han considerado el tabaquismo, dependencia de la nicotina, como un predictor positivo para el abuso y dependencia de otras sustancias psicoactivas $(11,16,29)$.

Un aspecto muy importante que es preciso considerar es la implicación del hábito de fumar y su relación causal con el carcinoma pulmonar (5). Algunos autores han expresado, por ejemplo, que los pacientes que padecen esquizofrenia tiene un riesgo global menor de presentar neoplasias que la población general (30); igualmente se ha afirmado que este factor protector está íntimamente relacionado con el efecto antineoplásico de los neurolépticos, que, en el caso del cáncer bronquioalveolar, esta menor incidencia estaría explicada por las altas concentraciones que alcanzan estas drogas en el tejido pulmonar (30).

Este trabajo tiene dos limitaciones: la primera, aunque no se catalogaron como fumadores los pacientes que fumaron alguna vez durante la adolescencia con carácter experimental, no se definió de manera rigurosa la categoría de fumador en relación con el tiempo del consumo y el número de cigarrillos por día; y, la segunda, no se consideró la procedencia de los pacientes, asumiendo que la totalidad residían en el departamento de Bolívar.

Se concluye que la prevalencia de tabaquismo en los pacientes que presentan un diagnóstico psiquiátrico es comparable a la de la población general, aunque es necesario resaltar que la prevalencia es mayor entre los pacientes con trastornos psicóticos y los que presentan trastornos relacionados con el consumo de sustancias. 
Sin duda, es necesario realizar un mayor número de estudios para precisar la relación entre los trastornos mentales y el hábito de fumar, como también determinar el riesgo de neoplasia pulmonar en pacientes psiquiátricos fumadores.

\section{Agradecimientos}

Al doctor Armando Morales, profesor titular, especializado en formulación y evaluación de proyectos y bioestadística del Departamento de Medicina Social, Universidad de Cartagena, por la asesoría estadística.

A la señora Josefina Castellar, auxiliar de biblioteca de la Universidad de Cartagena, por su gentil colaboración en la conmutación bibliográfica.

\section{Referencias}

1. Fielding JE. Smoking: health effects and control. $N$ Engl J Med 1985;313:491-8.

2. Cocores J. Nicotine dependence. Diagnosis and treatment. Psychiatr Clin North Am 1993;16:49-60.

3. Wolf PA. Cigarettes, alcohol and stroke. $N$ Engl $J$ Med 1986;315:1087-9.

4. Michnovicz JJ, Herhcopf RJ, Naganuma H, Bradlow HL, Fishman J. Increased 2-hydroxylation of estradiol as a possible mechanism for the anti-estrogenic effect of cigarette smoking. N Engl J Med 1986;315:1305-9.

5. Medina E, Denegri M, Donoso AM, Segovia E, Sierra A. Características del tabaquismo en enfermos hospitalizados. Rev Méd Chile 1987;115:584-7.

6. Ministerio de Salud. Estudio nacional de salud mental y consumo de sustancias psicoactivas (departamento de Bolívar). Bogotá: Ministerio de Salud, 1994:15.

7. Fiore MC. Tendencias en el tabaquismo de cigarrillo en Estados Unidos. Clin Méd Norte Am 1992;2:273-88.

8. Pierce JP, Fiore MC, Novotny TE, Hatziandreu EJ, Davis RM. Trends in cigarette smoking in the United States. Educational differences are increasing. JAMA 1989;261:56-60.

9. Kaplan HI, Sadock BJ, Grebb JA. Synopsis of psychiatry. 7th. edition. Baltimore: Williams \& Wilkins, 1994: 436-9.

10. Glass RM. Blue blood, blackened lungs. Depression and smoking (editorial). JAMA 1990;264:1583-4.

11. Meeks JE. Prevalence of chemical dependency in hospitalized general psychiatric patients (letter). Am J Psychiatry 1986;143:25-6.
12. Goff DC, Henderson DC, Amico E. Cigarette smoking in schizophrenia: relationship to psychopatology and medication side effects. Am J Psychiatry 1992;149: 1189-94.

13. McLellan AT, Woody GE, O'Brein CP. Development of psychiatric illness in drug abusers. N Engl J Med 1979; 301:1310-4.

14. Hughes JR, Hatsukami DK, Mitchell JE, Dahlgren LA. Prevalence of smoking among psychiatric outpatients. Am J Psychiatry 1986;993-7.

15. Glassman AH, Stetner F, Walsh T, Raizman PS, Fieiss JL, Cooper TB, et al.. Heavy smokers, smoking cessation and clonidine. Results of a double-blind, randomized trial. JAMA 1988;259:2863-6.

16. Breslau N, Kilbey MM, Andreski P. Nicotine dependence, major depression, and anxiety in young adults. Arch Gen Psychiatry 1991;48:1069-74.

17. De Leon J, Dadvand M, Canuso C, White AO, Stanilla JK, Simpson GM. Schizophrenia and smoking: an epidemiological survey in a state hospital. Am J Psychiatry 1995;152:453-5.

18. Dalack GW, Glassman AH, Rivelli S, Covey L, Stetner F. Mood, major depression, and fluoxetine response in cigarette smokers. Am J Psychiatry 1995; 152:398-403.

19. Glassman AH, Helzer JE, Covey LS, Cottler LB, Stetner F, Tipp JE, et al.. Smoking, smoking cessation, and major depression. JAMA 1990;264:1546-9.

20. Glassman AH. Cigarette smoking: implications for psychiatric illness. Am J Psychatry 1993;150:546-53.

21. Breslau N, Kilbey MM, Andreski P. Vulnerability to psychopathology in nicotine-dependent smokers: an epidemiologic study of young adults. Am $J$ Psychiatry 1993;150:941-6.

22. Flanagan J, Maany I. Smoking and depression (letter) Am J Psychiatry 1982;139:541.

23. Kendler KS, Neale MC, MacLean CL, Heath AC, Eaves LJ, Kessler RC. Smoking and major depression. A causal analysis. Arch Gen Psychiatry 1993;50:36-43.

24. Glassman AH, Jackson WK, Walsh BT, Roose SP. Cigarette craving, smoking withdrawal, and clonidine. Science 1984;226:864-6.

25. Hirschfeld RMA, Klerman GL, Clayton PJ, Keller M. Personality and depression. Arch Gen Psychiatry 1983; 993-8.

26. American Psychiatric Association. Diagnostic and statistical manual of mental disorders. 4th. edition. Washington,D.C.: American Psychiatric Association, 1994.

27. Kaplan HI, Sadock BJ. Manual de farmacoterapia en psiquiatría. Buenos Aires: Waverly Hispánica, 1993: 112-40. 
28. Campo A, Bermúdez A. Neurolépticos: consideraciones clínicas. Acta Méd Cartagena 1995;5:64-9.

29. Kozlowski LT, Wilkinson DA, Skinner W, Kent C, Franklin T, Pope M. Comparing tobacco cigarette dependence with other drug dependencies. Greater or equal "difficulty quitting' and "urges to use" but less "pleasure" from cigarette. JAMA 1989;261:898-901.

30. Masterson E, O'Shea B. Smoking and malignancy in schizophrenia. Br J Psychiatry 1984;145:429-32. 